\title{
Should medical students be given laparoscopic training?
}

\author{
Taner Shakir • Tae Lee • Jeffrey Lim • Kevin Jones
}

Received: 2 August 2014 / Accepted: 14 October 2014 / Published online: 9 November 2014

(C) Springer-Verlag Berlin Heidelberg 2014

\begin{abstract}
Undergraduate medical education does not usually involve training in laparoscopic skills despite the fact that minimal access surgery has become the norm in the developed world. We designed a study to evaluate the attitude of surgeons and medical students to formal teaching of these skills. Two surveys were sent; one to fourth year medical students at the University of Bristol and another to specialist laparoscopic surgeons. Student questions centred on whether they would find training in basic laparoscopic skills useful, whilst surgeons were asked whether it would be acceptable for medical students to assist with a laparoscopic case. Sixty percent [131/ 220] of students responded, with $60 \%$ [79/131] of respondents stating that they would find assisting with laparoscopic surgery beneficial, despite $79 \%$ [103/131] being undecided or having no interest in a surgical career, with $66 \%$ [87/131] stating it would allow them to become more involved during theatre sessions. Eighty-three percent [83/ 100] of surgeons responded, and $74 \%[62 / 83]$ said they would allow medical students to hold the camera. Seventy percent [65/83] felt that basic knowledge of the equipment was the most important aspect of training, and $66 \%$ [55/83] felt that assisting was the second most important. This is the first study to look at both the student's and surgeon's views on laparoscopic training of medical students. The study highlights the benefits of acquiring laparoscopic skills such as camera holding and assisting. In response, we have set up a course for students prior to placements in surgical specialties.
\end{abstract}

Electronic supplementary material The online version of this article (doi:10.1007/s10397-014-0870-4) contains supplementary material, which is available to authorized users.

T. Shakir · T. Lee $\cdot$ J. Lim $\cdot$ K. Jones $(\bowtie)$

University of Bristol Academy, Great Western Hospital, Marlbourgh

Road, Swindon SN3 6BB, UK

e-mail: kevin.jones@gwh.nhs.uk
Keywords Medical students · Laparoscopic skills training

\section{Introduction}

Medical student teaching in the operating theatre is often unsatisfactory [1]. The widespread introduction of laparoscopic surgery into clinical practice presents an opportunity to change this [2]. We believe that training in laparoscopic surgical techniques should start at medical school. Even if the student has no desire to pursue a surgical career after graduation, the opportunity to learn in the operating theatre will be enhanced if the student is taught basic laparoscopic techniques such as camera holding and the use of endoscopic graspers because they will be able to participate in the operation. In the past, students were asked to assist at operations by holding retractors so learning the basic techniques of laparoscopic surgery is no different in principle. Furthermore, being able to make a contribution in theatre will enhance the student's sense of belonging to the multidisciplinary team. They will no longer be asked to stand in a corner watching a TV monitor because they can once more "scrub in and join in".

In this study, we surveyed the attitude of medical students and experienced laparoscopic surgeons to the introduction of basic laparoscopic skills into the undergraduate medical curriculum. This is the first study of its kind in the UK.

\section{Objectives and methods}

We undertook a survey to investigate the attitude of surgeon's and students to introducing a laparoscopic training programme for medical students.

Two questionnaires were distributed electronically via email. The first (Appendix 1) questionnaire was sent to specialists in the UK and a second (appendix 2) questionnaire to 
fourth year medical students at Bristol University, UK. The specialists were asked eight questions, and one free text box was included. Six of the questions required a single answer, while two contained multiple stems with a choice of answers to semi quantify the specialists answer. The students were asked nine multiple choice questions (one answer only for each question) and one optional written answer question. In some of the questions and answers, bracket descriptions had to be included for clarity and specificity; otherwise, different interpretations and therefore different answers might be given that could affect the results of the study.

\section{Results}

All the students who responded were in the fourth year of their medical degrees (The Bristol medical degree is divided into two pre-clinical years and three clinical years). Sixty percent [131/220] of students responded, with $60 \%$ [79/131] of respondents stating that they would find assisting with laparoscopic surgery beneficial, despite $79 \%$ [103/131] being undecided or having no interest in a surgical career, with $66 \%$ [87/131] stating it would allow them to become more involved during theatre sessions. Seventy-one percent [93/ 131] of students would like to be offered a basic course in laparoscopic skills.

In total, $83 \%$ [83/100] of surgeons responded. Opportunities for student involvement are shown in Fig. 1a. There is an almost universal $98 \%$ acceptance of student observation. Seventy-four percent of surgeons were comfortable with students holding the camera, and $61 \%$ were comfortable with instrument holding whilst $51 \%$ would support retraction of tissues. Thirty-seven percent felt comfortable with medical students performing blunt dissection whilst $56 \%$ felt this should not be performed, and $65 \%$ felt that sharp should never be undertaken by students.

The skill specialists thought students could acquire are shown in Fig. 1b. Surgeons stated that basic knowledge of
Fig. 1 a Responses to" How comfortable would you be with medical students...? Lay observing includes non-scrubbed observation. Scrubbed observing includes close observation whilst sterile. b Responses to "How important are the following factors in allowing medical student involvement on your laparoscopic list? Basic surgical skills include suturing, etc. Basic knowledge of equipment entails knowledge of the stack and camera trouble shooting, e.g. lens fogging. Basic skills exercises are retraction with graspers and simple stacking exercises. Advanced camera skills are the use of a $30^{\circ}$ scope. Practice on a trainer includes active manipulation with graspers
A

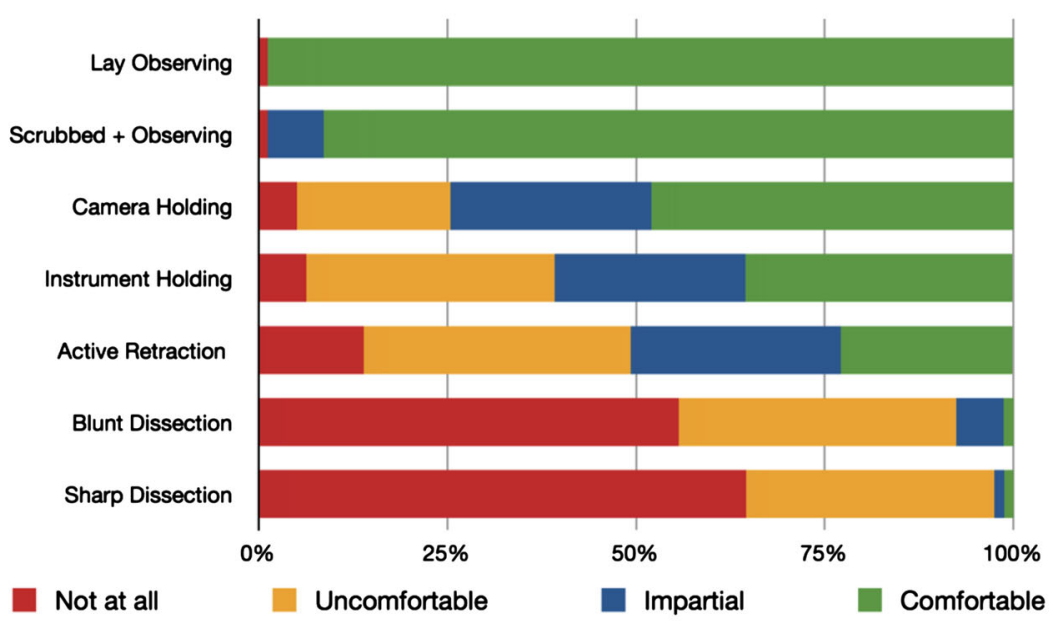

B

SKILLS

Basic knowledge of equipment

Basic skills exercises

Advanced camera skills

Practice on a trainer

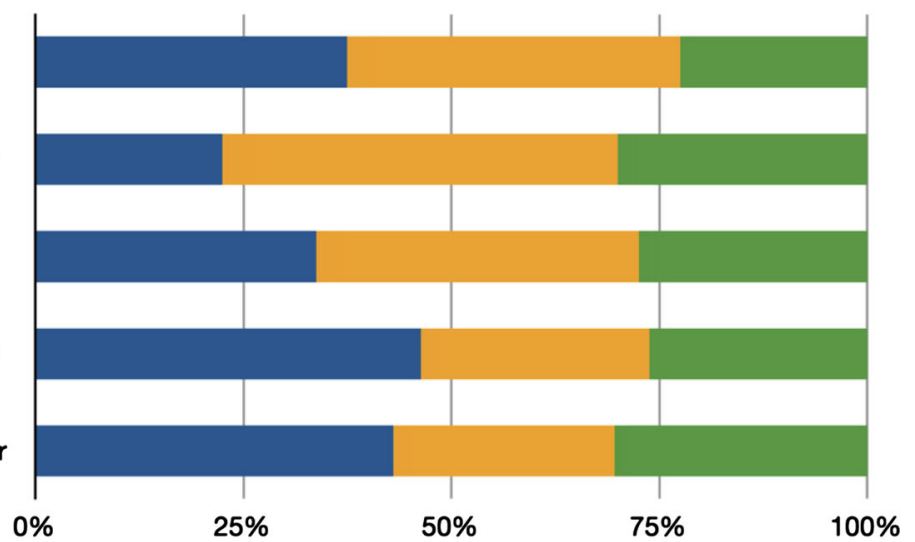

Not important
Essential 
the equipment was the most important (78 \%) [65/83], with basic laparoscopic skills being the second most important (66\%) [55/83] if such a course were to be offered to students.

\section{Discussion}

It is evident that an opportunity exists for medical students to be involved with laparoscopic procedures. This is not limited to camera holding, as over half were comfortable with instrument holding. A basic knowledge of the equipment in addition to basic skills exercises were the most important factors in allowing participation. This has implications for the development of a training programme.

Advantages to students are as follows:

\section{An Anatomy Lesson}

A decline in dissection at medical school has resulted in the current trend of increased utilisation of prosection, plastic models and even digital (virtual) methods for learning anatomy. These techniques can help students understand the relationships of structures to each other. However, laparoscopic surgery allows the student to have a "surgeon's eye view", so it should be viewed as an adjunct to the more modern approach of anatomy teaching.

\section{Involvement}

Medical students often feel as though theatre is not a favourable learning environment [1]. This translates into an uninspiring experience and may be a reason for the decline of interest in surgical careers [3]. Learning techniques such as camera and instrument holding offer the student an opportunity to participate in a low risk but essential task in theatre which in turn offers the chance for students to enhance their learning and enjoyment during undergraduate surgical placements.

Advantages to patients are as follows:

\section{Governance}

Laparoscopic techniques are usually learnt on trainers in a skills laboratory unlike open techniques which are first done on patients undergoing operations. The ability to acquire surgical skills in a simulated situation enhances patient safety and underpins modern concepts of good governance because it offers students an opportunity to practice with scope for error. Scenarios can be repeated indefinitely until trainees are confident. Teaching laparoscopic surgery in a simulated setting also allows core skills to be tested and measured.

2. The "Gameboy" generation

Patients can benefit from the changes already happening in society. Numerous systematic reviews have demonstrated a positive link between the acquisition of endoscopic skills and some video games [4-10]. Both laparoscopic surgery and video games require the translation of three-dimensional movement onto a two-dimensional screen. Concepts learned are "attentional weighting" (increased time spent on important aspects, whilst neglecting trivial features) as well as improving hand-eye coordination, dexterity and visuo-spatial abilities [4]. Video games are now so intrinsic to society (a norm even on mobile devices) that medical students may already possess these skills. Therefore, laparoscopy may be so intuitive to a modern-day medical student that it be natural. Observational studies have shown that age may also be a factor. In one study, there was a $77 \%$ improvement in completion time of basic laparoscopic skills for undergraduate medical students compared to junior surgical trainees [11]. The younger the student, the fewer problems with technical issues such as the fulcrum effect or swiping left in order to move right on smartphones.

Possible limitations

\section{Surgical experience}

We have assumed that basic and intermediate laparoscopic surgical techniques are part of every surgeon's skill set and that specialists have reached a level of competence where they can supervise a medical student to act as an assistant. We have also assumed that seniors are trained to give structured feedback. Where this is not the case, it will limit the involvement of students and post-graduate (PG) trainees in theatre. Furthermore, surgery is an apprenticeship which requires the acquisition of skills over time. This may be possible for a PG trainee, but medical students spend only short periods of time on surgical attachments.

\section{Training programmes}

There are many PG programmes available in laparoscopic surgery which offer training on simulators, and similar courses are now available for medical student's, e.g. ICENI Centre at Colchester Hospital, UK (www. icenicentre.org). In order to deliver a structured training programme, it is necessary to have an evidence-based curriculum. The European Society of Gynaecological Surgery (ESGE) has, in collaboration with the European Academy of Gynaecological Surgery, developed such a curriculum [12-15]. The full programme is called Gynaecological Endoscopic Surgical Education and Assessment (GESEA, www.esge.org/education/guidlines/ gesea). This training opportunity is accessible to everyone including medical students. 


\section{Conclusions}

This is the first study to look at both the student's and surgeon's views on laparoscopic training of medical students. Both students and surgeons have highlighted the potential benefit of students acquiring basic skills such as camera holding. In light of these findings, we have set up a SelfSelected Component in Surgery. Students will take part in a laparoscopic training course before being placed in surgical specialties where these skills can be put into practice. We plan to evaluate the students' experience to determine whether they gain more from their time in the operating theatre having been on the course.

Conflict of interest statement The authors whose names are listed in this article confirm that they have no affiliations with or involvement in any organisation or entity with any financial or non-financial interest in this project.

\section{References}

1. Fernando $\mathrm{N}$ et al (2007) Undergraduate medical students' perceptions and expectations of theatre-based learning: how can we improve the student learning experience? Surgeon 5:271-274

2. Green CJ, Maxwell R, Verne J, Martin RM, Blazeby JM (2009) The influence of NICE guidance on the uptake of laparoscopic surgery for colorectal cancer. J Public Health 31(4):541-545

3. Polk H (1999) The declining interest in surgical careers, the primary care mirage, and concerns about contemporary undergraduate surgical education. Am J Surg 178:177-179
4. Lynch J, Aughwane P, Hammond TM (2010) Video games and surgical ability: a literature review. J Surg Educ 67:184189

5. Rosenberg BH, Landsittel D, Averch TD (2005) Can video games be used to predict or improve laparoscopic skills? J Endourol 19:372 376

6. Fanning J, Fenton B, Johnson C, Johnson J, Rehman S (2011) Comparison of teenaged video gamers vs PGY-I residents in obstetrics and gynecology on a laparoscopic simulator. J Minim Invasive Gynecol 18:169-172

7. Rosser JC et al (2007) The impact of video games on training surgeons in the 21st century. Arch Surg 142:181-186, Discusssion 186

8. Rosenberg BH, Landsittel D, Averch TD (2005) Can video games be used to predict or improve laparoscopic skills? J Endourol 19:372 376

9. Ou Y, McGlone ER, Camm CF, Khan OA (2013) Does playing video games improve laparoscopic skills? Int J Surg 11: 365-369

10. Waxberg SL, Schwaitzberg SD, Cao CGL (2005) Effects of videogame experience on laparoscopic skill acquisition. Proc Hum Factors Ergon Soc Annu Meet 49:1047-1051

11. Salkini MW, Hamilton AJ (2010) The effect of age on acquiring laparoscopic skills. J Endourol 24:377-379

12. Campo R, Molinas CR, De Wilde RL, Brolmann H, Brucker S, Mencaglia L, Odonovan P, Wallwiener D, Wattiez A (2012) Are you good enough for your patients? The European certification model in laparoscopic surgery. Facts Views Vis Obgyn 4:95-101

13. Campo R, Reising C, Van Belle Y et al (2010) A valid model for testing and training laparoscopic psychomotor skills. Gynecol Surg 7: 133-141

14. Molinas CR, Campo R (2010) Defining a structured training program for acquiring basic and advanced laparoscopic psychomotor skills in a simulator. Gynecol Surg 7:427-435

15. Campo R, Wattiez A, Leon De Wilde R, Molinas Sanabria CR (2012) Training in laparoscopic surgery: From the lab to the or. Slovenian. J Public Health. doi:10.2478/v10152-012-0032-x 